\title{
Análisis de la posición del Perú en los reportes de Competitividad del Foro Económico Mundial
}

Competitiveness of Peru: Analysis of Peru's position in the Competitiveness Reports of the World Economic Forum

Rosario del Pilar López Padilla ${ }^{1}$, Lino Rodríguez Alegre $^{2}$, Jaime Calva Moreyra ${ }^{2}$

\section{RESUMEN}

Objetivo: Analizar, mediante el modelo de regresión lineal los escenarios probables de la posición de nuestro país en el ranking del Foro Económico Mundial (WEF), para los periodos de 2008 - 2017 (escenario optimista) y del 2012 - 2017 (escenario pesimista). Métodos: Se ha analizado el comportamiento de las posiciones ocupadas por el Perú en el ranking de los Reportes de Competitividad del Foro Económico Mundial de los años 2008 al 2017 y que guardan correlato con el Índice de Competitividad Global (ICG) el cual que va desde 1 (poco competitivo) a 7 (muy competitivo). Se ha efectuado la proyección de las probables posiciones del Perú mediante el modelo de regresión lineal Resultados: Se encontró valores de $R^{2}$ de $27,45 \%$, en un escenario optimista y, $R^{2}$ $=87,47 \%$ en un escenario pesimista respectivamente. Conclusiones: La probabilidad de que se acentúe el descenso que se observa en las posiciones del país en dicho ranking se acentúa en la medida que no se lleven a cabo las reformas estructurales que son necesarias para darle impulso a la competitividad, el crecimiento sostenido del país. y la confianza de los agentes económicos.

Palabras clave: Competitividad, Perú, Desarrollo económico.

\section{ABSTRACT}

Objective: To analyze, by means of the linear regression model the probable scenarios of the position of our country in the ranking of the Economic World Forum (WEF) for the periods 2008 - 2017 (optimistic scenario) to 2012 - 2017 (pessimistic scenario). Methods: There has been analyzed the behavior of the positions occupied by Peru in the ranking of the Reports of Competitiveness of the Economic World Forum from the year 2008 to 2017. The positions occupiAed by our country are correlated with the Index of Global Competitiveness (ICG) which goes from 1 (slightly competitive) to 7 (very competitive). There has been made the projection of the probable positions of Peru by means of the linear regression model. Results: It has been found a $R^{2}=27,45 \%$ value for an optimistic scenario and $\mathrm{R}^{2}=87,47 \%$ value for a pessimistic scenario. Conclussions: The probability of an accentuated the decrease that is observed in the positions of the country in ranking will continue if there are not carried out the structural reforms that are necessary to impulse the competitiveness, the sustained growth and the confidence of the economic agents.

Keywords: Competitivenes, Perú, economic development. 


\section{INTRODUCCIÓN}

En el Reporte de Competitividad Global del Foro Económico Mundial (WEF) para el periodo 2017-2018, que recoge la información acerca de la competitividad de 137 naciones, el Perú se ubica en la posición 72, habiendo retrocedido cinco lugares respecto al reporte del año 2016, donde ocupó el puesto 67 entre 138 países. Se confirma así el sostenido retroceso en la competitividad del país desde el ranking del año 2013 donde ocupó la posición 61 entre 148 países.

La importancia de la competitividad radica en el hecho de que los elementos que la configuran son fundamentales y determinantes tanto para el crecimiento de los países; así mismo están relacionad con el aumento de la productividad y contribuyen a hacer atractivo al país en términos de incentivar la inversión extranjera.

De acuerdo con información proveniente del The Global Competitiveness Report World Economic Forum (2017 -2018), el modelo de competitividad del WEF toma en su construcción factores macro y microeconómica y se elabora a partir de la información que recoge donde se muestra el ordenamiento de los países según el Índice de Competitividad Global (ICG). Este en una escala que va desde 1 (poco competitivo) a 7 (muy competitivo).

El índice de competitividad global del WEF, introducido el año 2005, se sustenta en 12 pilares los cuales determinan los indicadores de desempeño: (Figura 1).

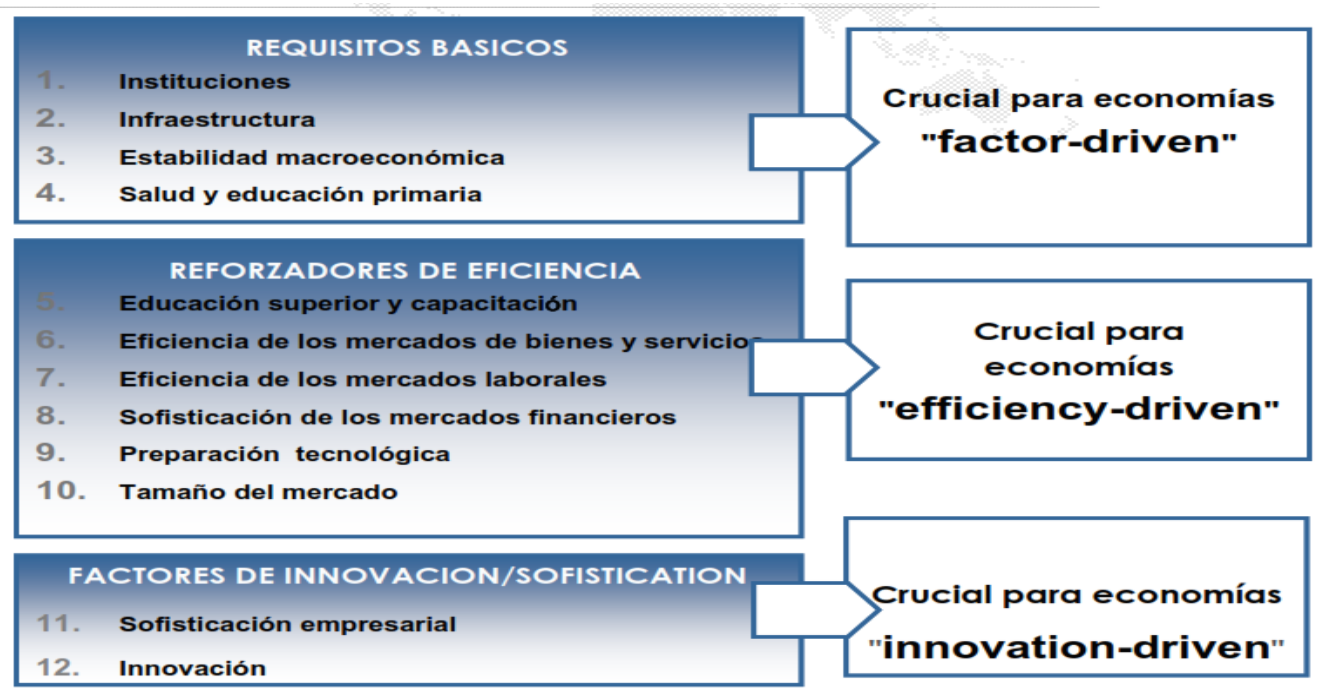

Fuente: World Economic Forum

Figura 1. Índice de Competitividad Global

Los 12 pilares de competitividad y los 114 indicadores primarios que recogen estos pilares, están organizados en 3 subíndices. La importancia de cada pilar dependerá de la etapa de desarrollo en la que este se encuentre el país. Los subíndices corresponden al detalle siguiente:

* “Factor-driven stage": En esta etapa, las empresas compiten en términos de precios, aprovechando los factores de producción baratos que están a su alcance. Aquí el sub índice referido a los requisitos básicos son cruciales para los países en esta etapa de desarrollo.

$\checkmark$ "Efficiency-driven stage": Se caracteriza por prácticas de producción eficientes para aumentar la productividad. Aquí los factores 
relacionados con los Reforzadores de eficiencia cobran protagonismo para los países que se ubican en este estadío de desarrollo.

$\checkmark$ "Innovation-driven stage": Las economías necesitan generar productos innovadores, utilizando métodos de producción sofisticados aprovechando plenamente las Tecnologías de Información y Comunicaciones (TICs). El protagonismo está en los factores de sofisticación e innovación. En esta agrupación se ubican los países de mayor desarrollo y que son los más competitivos. El índice de competitividad del WEF agrupa a los países en 5 estadíos de desarrollo.

Etapa I están los países de baja competitividad ("factor driven stage"),

$\checkmark$ Etapa II: agrupa a los países de competitividad media-baja (en transición de la etapa I a la etapa II),

$\checkmark$ Etapa III: aquellos de competitividad media ("Efficiencydriven stage"),
Etapa IV: los países de competitividad media - alta (transición de la etapa III a la etapa IV), y

$\checkmark$ Etapa $V$ : aquellos países de alta competitividad ("Innovation-driven stage")

En la estimación del índice competitividad el modelo del WEF establece ponderaciones de los tres subíndices para cada etapa de desarrollo (Tabla 1). Respecto a las fuentes que alimentan el cálculo del índice, los datos cuantitativos provienen de instituciones internacional como el Banco Mundial, el FMI, entre otros, así como de las encuestas de opinión ejecutiva a líderes empresariales a nivel mundial quienes son cuidadosamente seleccionados en cada país, a fin de reflejar de la mejor manera la estructura del ambiente empresarial nacional. La información provista por estas encuestas, son indispensables, en particular, para aquellas variables donde no se tienen fuentes fiables de información de índole cuantitativa.

Tabla 1. Ponderaciones de las etapas de desarrollo de los países

\begin{tabular}{l|ccc|}
\hline & $\begin{array}{c}\text { Factor - driven } \\
\text { stage }\end{array}$ & $\begin{array}{c}\text { Efficiency - } \\
\text { driven stage }\end{array}$ & $\begin{array}{c}\text { Innovation - } \\
\text { driven stage }\end{array}$ \\
\hline Basic requirements & $60 \%$ & $40 \%$ & $20 \%$ \\
\hline Efficieny enhancers & $35 \%$ & $50 \%$ & $50 \%$ \\
\hline $\begin{array}{l}\text { Innovation and } \\
\text { sophistication factors }\end{array}$ & $5 \%$ & $10 \%$ & $30 \%$ \\
\hline
\end{tabular}

Fuente: The Global Competitiveness Report 2017 -2018 World Economic Forum.

El modelo del índice de competitividad del WEF está basado en las concepciones teóricas de Michael Porter (1991), propuestas en su libro La Ventaja competitiva de las Naciones, donde señala que la ventaja competitiva de las naciones del siglo xxi sería creada y no heredada.

El modelo ayuda a comprender el éxito de algunos países en aumentar sus niveles de ingresos ampliando el bienestar y las oportunidades para sus poblaciones siendo, además, una herramienta de referencia para formular políticas económicas y reformas institucionales para quienes desarrollan políticas de estado ofreciendo, también, a los empresarios las pistas necesarias para tomar sus decisiones estratégicas y de inversión. 
$\begin{array}{ccc}\text { Saldarriaga } & \text { (2016), señala la } \\ \text { importancia del Indice de }\end{array}$ Competitividad Global (ICG) pues identifica las principales áreas donde son necesarias mejoras para elevar el nivel de competitividad pues está positivamente asociado a la productividad total de factores y al nivel de PBI per cápita.

Sala-i - Martin et al, (2014), señala que el Foro Económico Mundial (WEF) define la competitividad como "el conjunto de instituciones, políticas $y$ factores que determinan el nivel de productividad de un país. El nivel de productividad a su vez, determina el nivel de prosperidad que puede ser alcanzado por una economía"

Porter, M (1991), refiere que la prosperidad de un país depende de su competitividad y esta: "...se basa en la productividad con la cual el país produce sus bienes y servicios". Respecto a los mecanismos que sientan las bases de la misma, Porter señala que: "Se requieren políticas macroeconómicas sólidas e instituciones legales y políticas estables, pero estas no son suficientes para asegurar que una economía sea próspera pues la competitividad está enraizada en los fundamentos microeconómicos - la sofisticación de las operaciones $y$ las estrategias empresariales y la calidad del entorno microeconómico de negocios en el que las empresas compiten."

Indacochea et al, (1998), refiriéndose al modelo del "diamante de competitividad" de Porter señala que este posibilita comprender el proceso que lleva al logro de ventajas competitivas internacionales, así como identificar la manera cómo los países pueden desarrollar su competitividad; esto es: mejorar la cantidad y calidad de los recursos que poseen y la capacidad para utilizar esos recursos más eficientemente.
Según el modelo de Porter M. (1991), el que una nación cuente con sectores capaces de competir exitosamente en el mercado internacional depende en gran medida del contexto que rodea a las empresas que conforman cada sector, pues éstas no son entes aislados. El entorno nacional al que hace referencia Porter, está determinado por la interrelación de cuatro grupos de atributos:

$>$ Las condiciones de la demanda. Trata de la naturaleza de la demanda interna o los servicios del sector.

$>$ Sectores afines y de apoyo. Es decir, industrias proveedoras y relacionadas que pueden formar clusters.

$>$ Las condiciones de los factores. Se refiere a la mano de obra especializada 0 infraestructura; esto es, los factores de producción del país.

$>$ La estrategia, estructura y rivalidad de las empresas. Son las condiciones que conciernen a la creación, organización y gestión de las compañías.

Además, el modelo del diamante de Porter, precisa que existen dos variables adicionales que se incluyen en esta teoría: el azar y el Gobierno como elementos que pueden influir de manera importante en las industrias.

Articulando lo anterior, tenemos pues que la competitividad de un país es la capacidad de producir bienes y servicios con valor qué puestos a satisfacer necesidades, permitan generar condiciones de bienestar y prosperidad para todos sus ciudadanos; sin embargo, en el caso del país, a pesar de los logros macroeconómicos, pareciera que hemos caído en la autocomplacencia.

Seguimos catalogados como perteneciente al bloque de los países con ingresos medios y no logramos salir 
de la "trampa de ingresos medios" Situación en la cual un país emergente entra en un estancamiento que detiene su convergencia hacia los niveles de ingreso de los países desarrollados, el PBI per cápita PPP del Perú, ajustado a dólares constantes del 2013, es de US \$ $11,800.00$ (Banco Mundial, 2013), por la falta de las reformas necesarias que posibiliten un desarrollo integral, sostenido y duradero elevando la productividad.

Salir de la misma obliga a la mejora de la competitividad del país y de las empresas y ello va asociado con elevar la productividad. Para esto debemos tener metas nacionales claras, liderazgo del Gobierno Central y compromiso del sector público y privado donde el fomento del crecimiento basado en la innovación sea la fuente.

Lograr el despegue lleva a mantener el foco en reformas de mediano y largo plazo que van asociadas a mejoras en variables como: infraestructura, capital humano, institucionalidad e innovación y donde tenemos brechas evidentes. Podría decirse que estamos pagando las consecuencias de no haber hecho reformas en los últimos 10 años.

La turbulencia política interna de los últimos años sumados a los casos de corrupción que involucra inclusive, a quienes toman decisiones a nivel macro, sumados a la falta de profundización de las reformas han impedido la implementación de políticas e instrumentos que contribuyan al fomento del flujo de inversiones que puedan atraer capitales de largo plazo que promuevan la competitividad.

Michael Porter, en el "Seminario Internacional: Claves de una Estrategia Competitiva" organizado por la Universidad del Pacífico e Interbank diciembre 2009, señalaba que "El Perú atraviesa tiempos económicamente peligrosos pues las fuerzas que explican el crecimiento reciente no son sostenibles porque no generan competitividad. Perú depende demasiado de las exportaciones de materias primas, que tienen un potencial limitado para impulsar el crecimiento económico y la diversificación económica y aunque se había logrado progresos en política macroeconómica no se estaba invirtiendo lo suficiente en recursos humanos e infraestructura $y$ que se debía cambiar la estructura productiva y pasar de ser un sector primario a uno industrializado con valor agregado y generación de empleos pues las exportaciones de materias primas tienen un potencial limitado para impulsar el crecimiento económico y la diversificación económica y no estamos invirtiendo lo suficiente en recursos humanos y la inversión en innovación y desarrollo".

Los modelos de desarrollo del país han priorizado aprovechar las ventajas comparativas basadas en la explotación de los recursos naturales y la mano de obra barata; por ejemplo: exportar los minerales o la riqueza de nuestro mar. En la era del conocimiento, el capital humano es el factor estratégico de competitividad y los países deben dar paso a las ventajas competitivas; aquellas que se crean como producto de la innovación y creatividad, la inversión en capital humano, la formación profesional de calidad y el impulso a la investigación y desarrollo.

Finalmente, Alvarez Rodrich, (2009) comentando la presentación de Porter señala que "debemos avanzar en ciencia y tecnología. Esto se logra con mejoras en la calidad de la educación y salud, mejorar la infraestructura $y$ avanzar en la reducción de la pobreza; lo que implica dar el salto como país, a la modernidad y pasar de la categoría de los países que solo crecen a la de aquellos que progresan como nación como Singapur, Taiwán o Nueva Zelanda y que este es un proceso que se puede conseguir en alrededor de 
cuatro décadas haciendo las cosas consistentemente bien mediante reformas profundas en educación, salud, seguridad, justicia, organización del sector público y del servicio civil, y la competitividad de las empresas y de la sociedad en su conjunto. Una agenda pendiente desde hace tiempo y que ningún presidente peruano de por 10 menos el último medio siglo ha tenido el coraje de emprender".

\section{MATERIAL Y MÉTODOS}

Se analizo, el comportamiento de las posiciones ocupadas por el Perú en el Ranking de competitividad global publicado en los Reporte de Competitividad Global del WEF del periodo 2008 al 2017 del WEF las mismas que se recoge en la Tabla 2.

Tabla 2. Ranking de competitividad global del Perú Periodos (2007-2017)

\begin{tabular}{|l|c|c|}
\hline & Puesto & Puntaje \\
\hline GCI 2017 -2018 (total 137 países) & 72 & 4,2 \\
\hline GCI 2016 -2017 (total 138 países) & 67 & 4,2 \\
\hline GCI 2015-2016 (total 140 países) & 69 & 4,2 \\
\hline GCI 2014 -2015 (total 144 países) & 65 & 4,2 \\
\hline GCI 2013 -2014 (total 148 países) & 61 & 4,3 \\
\hline GCI 2012 -2013 (total 144 países) & 61 & 4,3 \\
\hline GCI 2011 -2012 (total 142 países) & 67 & 4,2 \\
\hline GCI 2010 -2011 (total 139 países) & 73 & 4,1 \\
\hline GCI 2009 -2010 (total 133 países) & 78 & 4,0 \\
\hline GCI 2008 -2009 (total 134 países) & 83 & 3,9 \\
\hline GCl 2007-2008 (total 131 países) & 86 & 3,9 \\
\hline
\end{tabular}

Fuente: World Economic Forum Global Competitiveness Reports 2007 -2017

La Tabla 3 muestra el comparativo, entre el puntaje obtenido por cada uno de los indicadores de los pilares de competitividad y la posición ocupada por el Perú en el ranking para el periodo de análisis.

Tabla 3. Comparativo entre la puntuación y la posición de los indicadores de los pilares de competitividad - Ranking de competitividad global WEF-Perú (periodo 2008-2017)

\begin{tabular}{|c|c|c|c|c|c|c|c|c|c|c|}
\hline Índice de Competividad Global & 2008-2009 & PUNTAJE & $\begin{array}{l}2009- \\
2010 \\
\end{array}$ & PUNTAJE & $\begin{aligned} 2010- \\
2011 \\
\end{aligned}$ & PUNTAJE & $\begin{array}{ll}2011- \\
2012 \\
\end{array}$ & PUNTAJE & $\begin{array}{l}2012- \\
2013\end{array}$ & PUNTAJE \\
\hline Posición del Perú & 83 & 3,9 & 78 & 4,0 & 73 & 4,1 & 67 & 4,2 & 61 & 4,3 \\
\hline Requerimientos básicos & 94 & 4,0 & 88 & 4,1 & 87 & 4,2 & 78 & 4,4 & 69 & 4,6 \\
\hline Instituciones & 101 & 3,5 & 90 & 3,5 & 96 & 3,5 & 95 & 3,5 & 105 & 3,4 \\
\hline Infraestructura & 110 & 2,5 & 97 & 2,9 & 88 & 3,5 & 88 & 3,6 & 89 & 3,5 \\
\hline Estabilidad Macroeconómica & 67 & 5,0 & 63 & 4,7 & 75 & 4,5 & 52 & 5,0 & 21 & 5,9 \\
\hline Salud y Educación primaria & 95 & 5,1 & 91 & 5,1 & 92 & 5,4 & 97 & 5,4 & 91 & 5,4 \\
\hline Reforzadores de la eficiencia & 69 & 4,0 & 59 & 4,1 & 56 & 4,2 & 50 & 4,3 & 57 & 4,2 \\
\hline $\begin{array}{l}\text { Educación superior y capacitación } \\
\text { Eficiencia del mercado de bienes }\end{array}$ & $\begin{array}{l}89 \\
61\end{array}$ & $\begin{array}{l}3,6 \\
4,2\end{array}$ & $\begin{array}{l}81 \\
66\end{array}$ & $\begin{array}{l}3,7 \\
4,2\end{array}$ & $\begin{array}{l}76 \\
69\end{array}$ & $\begin{array}{l}4,0 \\
4,2\end{array}$ & $\begin{array}{l}77 \\
50\end{array}$ & $\begin{array}{l}4,0 \\
4,4\end{array}$ & $\begin{array}{l}80 \\
53\end{array}$ & $\begin{array}{l}4,0 \\
4,4\end{array}$ \\
\hline $\begin{array}{l}\text { Eficiencia del mercado laboral } \\
\text { Sofisticación del mercado financiero }\end{array}$ & $\begin{array}{l}75 \\
45\end{array}$ & $\begin{array}{l}4,3 \\
4,7\end{array}$ & $\begin{array}{l}77 \\
39\end{array}$ & $\begin{array}{l}4,3 \\
4,7\end{array}$ & $\begin{array}{l}56 \\
42\end{array}$ & $\begin{array}{l}4,5 \\
4,6\end{array}$ & $\begin{array}{l}43 \\
38\end{array}$ & $\begin{array}{l}4,6 \\
4,5\end{array}$ & $\begin{array}{l}45 \\
45\end{array}$ & $\begin{array}{l}4,6 \\
4,5\end{array}$ \\
\hline Preparación tecnológica & 87 & 3,0 & 77 & 3,4 & 74 & 3,5 & 69 & 3,6 & 83 & 3,6 \\
\hline Tamaño del mercado & 50 & 4,2 & 46 & 3,4 & 48 & 4,3 & 48 & 4,3 & 45 & 4,4 \\
\hline Factores de innovación & 83 & 3,4 & 85 & 3,4 & 89 & 3,3 & 89 & 3,3 & 108 & 3,3 \\
\hline Sofisticación empresarial & 67 & 4,1 & 68 & 4,0 & 71 & 3,8 & 65 & 3,9 & 78 & 3,9 \\
\hline
\end{tabular}




\begin{tabular}{|c|c|c|c|c|c|c|c|c|c|c|c|}
\hline Innovación & 110 & & & &, 7 & 10 & 2,7 & 113 & 27,0 & 119 & 2,7 \\
\hline Total de economías en la muestra & & 134 & & 133 & & 139 & & 142 & & & 44 \\
\hline Índice de Competividad Global & 2013- 2014 & PUNTAJE & 2014-2015 & PUNTAJE & 2015-2016 & PUNTAJE & 2016-2017 & PUNTAJE & $\begin{array}{l}2017- \\
2018\end{array}$ & PUNTAJE & VARIACIÓN \\
\hline Posición del Perú & 61 & 4,3 & 65 & 4,2 & 69 & 4,2 & 67 & 4,2 & 72 & 4,2 & -5 \\
\hline Requerimientos básicos & 72 & 4,5 & 74 & 4,5 & 76 & 4,5 & 77 & 4,4 & 79 & 4,4 & -2 \\
\hline Instituciones & 109 & 3,4 & 118 & 3,3 & 116 & 3,3 & 106 & 3,4 & 116 & 3,2 & -10 \\
\hline Infraestructura & 91 & 3,5 & 88 & 3,5 & 89 & 3,5 & 89 & 3,6 & 86 & 3,8 & 3 \\
\hline Estabilidad Macroeconómica & 20 & 5,9 & 21 & 5,9 & 23 & 5,9 & 33 & 5,4 & 37 & 5,4 & -4 \\
\hline Salud y Educación primaria & 95 & 5,4 & 94 & 5,4 & 100 & 5,3 & 98 & 5,3 & 93 & 5,4 & 5 \\
\hline Reforzadores de la eficiencia & 57 & 4,2 & 62 & 4,2 & 60 & 4,2 & 57 & 4,3 & 64 & 4,2 & -7 \\
\hline $\begin{array}{l}\text { Educación superior y capacitación } \\
\text { Eficiencia del mercado de bienes }\end{array}$ & $\begin{array}{l}86 \\
52\end{array}$ & $\begin{array}{l}4,0 \\
4,4\end{array}$ & $\begin{array}{l}83 \\
53\end{array}$ & $\begin{array}{l}4,1 \\
4,5\end{array}$ & $\begin{array}{l}82 \\
60\end{array}$ & $\begin{array}{l}4,1 \\
4,4\end{array}$ & $\begin{array}{l}80 \\
65\end{array}$ & $\begin{array}{c}4,1 \\
44,0\end{array}$ & $\begin{array}{l}81 \\
75\end{array}$ & $\begin{array}{l}4,1 \\
4,3\end{array}$ & $\begin{array}{c}-1 \\
-10\end{array}$ \\
\hline $\begin{array}{l}\text { Eficiencia del mercado laboral } \\
\text { Sofisticación del mercado financiero }\end{array}$ & $\begin{array}{l}48 \\
40\end{array}$ & $\begin{array}{l}4,5 \\
4,5\end{array}$ & $\begin{array}{l}51 \\
40\end{array}$ & $\begin{array}{l}4,3 \\
4,5\end{array}$ & $\begin{array}{l}64 \\
30\end{array}$ & $\begin{array}{l}4,3 \\
4,5\end{array}$ & $\begin{array}{l}61 \\
26\end{array}$ & $\begin{array}{l}4,3 \\
4,7\end{array}$ & $\begin{array}{l}64 \\
35\end{array}$ & $\begin{array}{l}4,3 \\
4,5\end{array}$ & $\begin{array}{l}-3 \\
-9\end{array}$ \\
\hline Preparación tecnológica & 86 & 3,4 & 92 & 3,3 & 88 & 3,4 & 88 & 3,6 & 86 & 3,7 & 2 \\
\hline Tamaño del mercado & 45 & 4,5 & 43 & 4,5 & 48 & 4,4 & 48 & 4,4 & 48 & 4,5 & 0 \\
\hline Factores de innovación & 97 & 3,4 & 99 & 3,3 & 106 & 3,3 & 108 & 3,3 & 103 & 3,3 & 5 \\
\hline Sofisticación empresarial & 74 & 3,9 & 72 & 3,9 & 81 & 3,8 & 78 & 3,8 & 80 & 3,8 & -2 \\
\hline Innovación & 122 & 2,8 & 117 & 2,8 & 116 & 2,8 & 119 & 2,8 & 113 & 2,8 & 6 \\
\hline Total de economías en la muestra & \multicolumn{2}{|c|}{148} & \multicolumn{2}{|c|}{144} & \multicolumn{2}{|c|}{140} & \multicolumn{2}{|c|}{138} & \multicolumn{2}{|c|}{137} & 1 \\
\hline
\end{tabular}

Fuente: Elaboración propia de los Reportes de Competitividad Global del WEF 2008 - 2017

La data anterior ha sido la fuente de referencia para analizar el desempeño de la competitividad del país.

Mediante el modelo de regresión lineal se han modelado dos escenarios de desempeño futuro, en términos de la posición de nuestro país en el ranking del Foro Económico Mundial, para los periodos de 2018 al 2022. A estos escenarios se les ha denominado como optimista y pesimista respectivamente. De modo complementario los resultados de la Tabla 3 nos permiten hacer el análisis de las razones que explican las posiciones ocupadas por el país en dicho ranking y las mejoras en las que debe incidir.

\section{RESULTADOS}

Según el ranking de Ranking de competitividad global WEF, para el año 2017, el Perú ha descendió 5 posiciones con relación a la posición ocupada en el año anterior. Esto es hemos pasado del puesto 67 al 72 . Con ello se confirma la tendencia al descenso que se observa en el ranking desde el año 2013 como se aprecia en la Figura 2

\section{Posicion del peru en el ranking de competitividad}

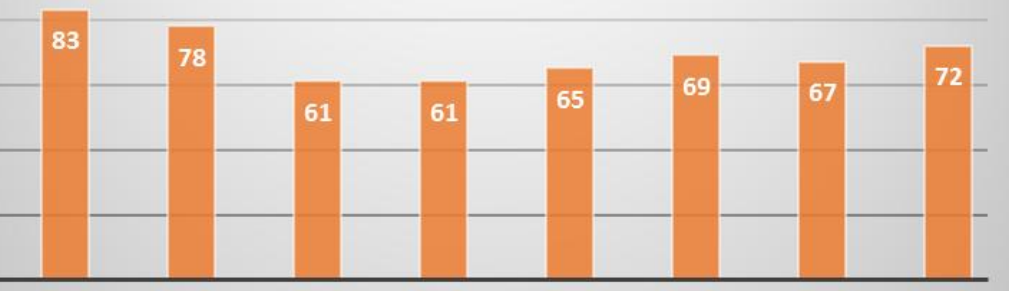

2008 -2009 2009-2010 2012-2013 2013-2014 2014-2015 2015-2016 2016-2017 2017-2018

$$
\text { - Pilares } \text { - Posición del Perú }
$$


Figura 2. Posición del Perú en el ranking de competividad

Se han modelado, mediante regresión lineal simple, los escenarios probables de la posición de nuestro país en el ranking del Foro Económico Mundial, para los periodos de $2008-2017$ y del 2012 - 2017. A estos se le ha denominado como escenario optimista y otro pesimista, respectivamente.

Respecto al escenario optimista, que comprende la información de los años 2008 al 2017, muestra en la Tabla 4 y Figura 3 correspondiente, tenemos que el país podría escalar posiciones hasta el lugar 58. Sin embargo, basado en el $\mathrm{R}^{2}$ de la regresión, la ocurrencia de esta posibilidad es de sólo el 27,45\%.

Respecto al escenario pesimista, dado que la data histórica muestra una tendencia a la baja, desde el 2012, los valores obtenidos se aprecian en la Tabla 5 y Figura 4 respectivamente.

El descenso en las posiciones del ranking podría, en el extremo hasta llegar el lugar 91 ; y como lo indica el $\mathrm{R}^{2}$ de la tendencia de regresión con una posibilidad ocurrencia del $87,47 \%$.

Tabla 4. Escenario optimista y pronóstico de tendencias

\begin{tabular}{lc}
\hline \multicolumn{2}{c}{ Escenario Optimista } \\
\hline $\mathrm{a}=$ & 76,333 \\
$\mathrm{~b}=$ & $-1,2242$ \\
$\mathrm{R} 2=$ & $27,45 \%$ \\
\hline
\end{tabular}

\begin{tabular}{ccc}
\hline \multicolumn{3}{c}{ Pronóstico De Tendencias } \\
Años & Periodo & Posición \\
\hline 2018 & 11 & $\mathbf{6 3}$ \\
2019 & 12 & $\mathbf{6 2}$ \\
2020 & 13 & $\mathbf{6 0}$ \\
2021 & 14 & $\mathbf{5 9}$ \\
2022 & 15 & $\mathbf{5 8}$ \\
\hline
\end{tabular}

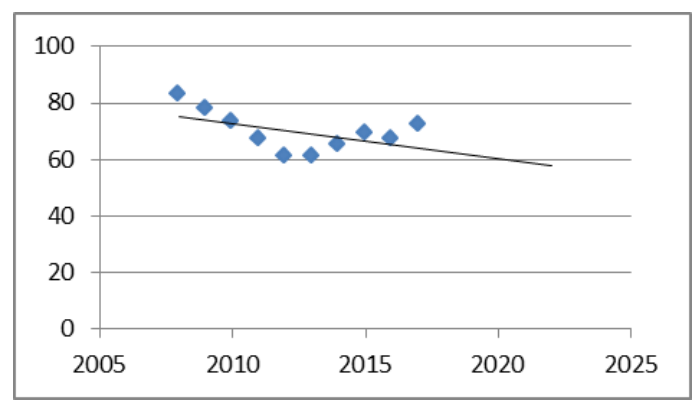

Figura 3. Escenario optimista

Tabla 5. Escenario pesimista y pronóstico de tendencias

\begin{tabular}{lc}
\hline \multicolumn{2}{c}{ Escenario Pesimista } \\
\hline $\mathrm{a}=$ & 58,13 \\
$\mathrm{~b}=$ & 2,2 \\
$\mathrm{R} 2=$ & $87,47 \%$ \\
\hline
\end{tabular}

\begin{tabular}{ccc}
\hline \multicolumn{3}{c}{ Pronóstico De Tendencias } \\
Años & Periodo & Posición \\
\hline 2018 & 11 & 82 \\
2019 & 12 & 85 \\
2020 & 13 & 87 \\
2021 & 14 & 89 \\
2022 & 15 & 91 \\
\hline
\end{tabular}




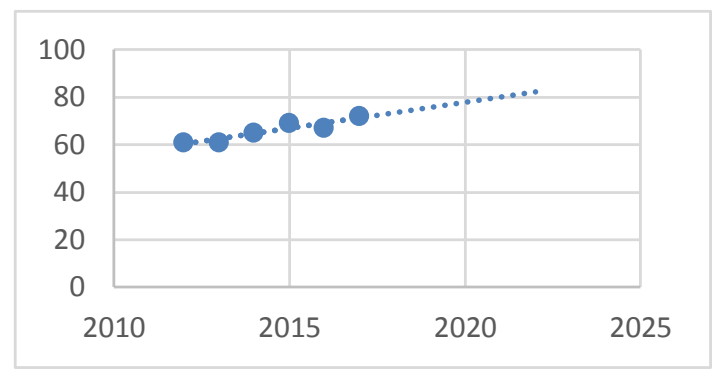

Figura 4. Escenario pesimista

\section{DISCUSIÓN}

El problema es que los esfuerzos del país habrían menguado en este periodo de 2012 a 2017, seguramente se ha procurado sostener las posiciones expectantes anteriores pero muy poco se habría hecho por avanzar, permitiendo que otros países logren escalar posiciones por encima de la nuestra. Pero lo más importante es que esto nos indica tomar medidas como la profundización de las reformas estructurales, las mismas que se han paralizado por la turbulencia política en una atmósfera de inestabilidad que afecta la confianza de los agentes económicos. Otro aspecto relevante, es la pérdida de credibilidad ciudadana en las instituciones del Estado en el marco de una efectiva lucha contra la corrupción; y la falta de medidas estratégicas en el plano económico para promover la diversificación productiva y la adición de valor agregado.

\section{REFERENCIAS BIBLIOGRÁFICAS}

Álvarez, Rodrich (2009, Diciembre, 08). "El portero llama dos veces". Diario La República.

Indacochea, A., Avolio, B., Bedoya, L., Carrillo, J., Negron, G., Sánchez, L., \& Santillana, M. (1998). Cajamarca competitiva. Saywa Ediciones SRL Lima.

Krugman, P. (1994). The age of diminishing expectations: US Economic Policy in the 1990s. Massachusetts: $\quad$ MIT Press Cambridge.

Porter, M. E. (1991). La ventaja competitiva de las naciones. Buenos Aires: Vergara.

Porter, M. (2009, Noviembre). Seminario internacional: claves de una estrategia competitiva. Ponencia presentada en el Seminario internacional realizado por la Escuela de Posgrado de la Universidad del Pacífico e INTERBANK, Lima, Perú.

Sala-i-Martin, X., Bilbao-Osorio, B., Di Battista, A., Drzeniek Hanouz, M., Geiger, T., \& Galvan, C. (2014). The Global Competitiveness Index 20142015: accelerating a robust recovery to create productive jobs and support inclusive growth. The global competitiveness report, 2015, 352.

Saldarriaga, M. (2016). Perú: Reporte de Competitividad Global 2016. Revista Moneda, (168), 25-29.

Schwab, K., Porter, Xavier Sala-iMartín., \& Richard Samans (2017). The global competitiveness report 200172018. World Economic Forum Geneva. ISBN-13: 978-1944835-11-8 
Correo

rlopezp@ucv.edu.pe

electrónico: 